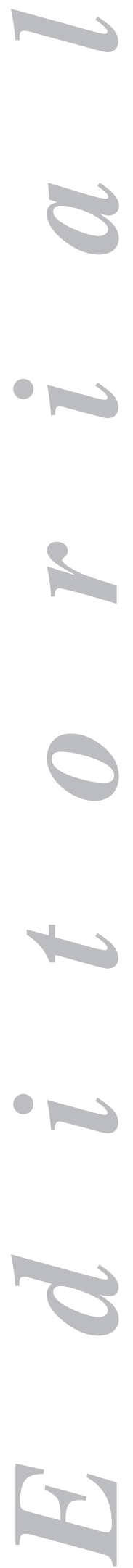

\title{
Intraductal papillary mucinous neoplasm of the pancreas: an emerging indication for surgical therapy
}

The extensive use of modern imaging modalities increasingly renders careful differential diagnosis a need when cystic profile lesions are found in the pancreas $(1,2)$. And of course intraductal papillary mucinous neoplasm of the pancreas must be carefully assessed in the differential diagnosis of such lesions. For every finding that is cystic in nature a first step is always to tell tumors, whether benign or malignant, from non-neoplastic conditions. To consider a cystic lesion neoplastic, the presence of an epithelial tissue band separating normal pancreatic tissue from the cyst must be identified.

In addition to pseudocyst, non-neoplastic cystic lesions of the pancreas include simple pancreatic cyst, a concept that remains poorly delimited. For some authors these non-neoplastic cysts would include retention cysts as well as mucoceles. However, other authors consider that smaller unilocular cysts with thin walls may similarly represent papillary tumors with low-grade dysplasia rather than the more concerning condition of intraductal papillary mucinous tumor with malignant changes.

Among neoplastic pancreatic cysts serous cystic tumors (cystadenoma and cystadenocarcinoma), mucinous cystic tumors (cystadenoma, cystadenoma with dysplasia, and mucinous cystadenocarcinoma), and intraductal papillary mucinous tumors (adenoma, tumor with dysplasia, and intraductal papillary mucinous carcinoma) should be considered, the latter being the subject of this editorial.

Serous cystic tumors most commonly involve the head of the pancreas and are typically multilocular with septa in their inside. Benign unilocular cystadenomas may on occasion exist that are preoperatively indistinguishable from unilocular mucinous cystic tumors.

Mucinous cystic tumors most commonly involve the body and tail of the pancreas, may be unilocular or multilocular, and typically affect women. They should be formally considered a lesion with a potential to progress from benignity to malignity. Multilocular tumors are easier to diagnose since walls are usually obvious and calcification may be present. However, unilocular lesions have a more challenging differential diagnosis. On occasion other non-cystic lesions such as pseudopapillary or endocrine tumors may undergo tissue necrosis resulting in a cyst, and thus should also be included in the differential diagnosis.

Intraductal papillary mucinous tumors originate in the tissue of pancreatic ducts, whether the main duct or any of its branches. Therefore, their diagnosis becomes more likely when the cystic tumor can be seen to communicate with pancreatic ducts at any level. These tumors are incidental findings in up to one third of cases.

Echoendoscopy increases diagnostic certainty in this type of neoplasm as in expert hands will allow a refined definition of lesion features and a guided puncture to 
collect fluid from the cyst, upon which both a cytological exam and tumor marker analysis will be performed $(1,3)$.

Both mucinous cystic tumors and intraductal papillary mucinous tumors should be considered surgical conditions. Regarding surgery resection may be deemed curative when lesion stage is confirmed to be prior to carcinoma. The probability that such a lesion has reached the carcinoma stage is currently estimated lower than $30 \%$ in such cases (2).

Laparoscopic pancreatic surgery may be indicated for these lesions in expert hands, provided they are not located at the head of the pancreas and the probability of potential transformation to oncologic surgery beyond cyst resection is reasonably low (4). Also in expert hands surgery risks are very low for mortality and low for morbidity, always in the absence of oncologic surgery beyond lesion resection, also with a low probability of sequels.

In the current issue of this journal we may find a number of case reports gathering the surgical experience of a relevant Spanish team. Cienfuegos et al. (5) provide us with the second most extensive series in the Spanish literature, with 13 patients operated on for intraductal papillary mucinous neoplasm. The primary objective of this manuscript is to assess said experience in line with international consensus meetings held in relation to this field (6-8). In this series oncological surgery predominated with no less than 4 total, 5 cephalic, 1 central and 1 distal pancreatectomies, and only two enucleations. Despite this operative mortality was nil. Three of the patients undergoing surgery had invasive cancer, and two had in situ carcinoma at the time of surgery. Patient follow-up oscillated from 1.5 months to 9 years, and notably only one died during follow-up, which clearly supports the fact that indications for surgery are appropriate. Such indications, as properly discussed by the authors, must be systematically envisaged except for cases where lesions are restricted to accessory pancreatic ducts, remain small in size, and exhibit no inner nodules.

Intraductal papillary mucinous neoplasm will become an increasingly common diagnosis where a high yield of aspiration puncture under echoendoscopic guidance may be expected, and where surgery should be used without hesitation, since progression in cases involving the main duct seem to slowly but inexorably evolve towards malignity. When excellent surgeons are available, as is the case with those sharing their experience in this issue, a high cure rate may be expected with negligible mortality and fairly acceptable morbidity.

F. Carballo-Álvarez

Service of Digestive Diseases. Hospital Universitario Virgen de la Arrixaca. Murcia, Spain

\section{REFERENCES}

1. Garcea G, Ong SL, Rajesh A, Neal CP, Pollard CA, Berry DP, et al. Cystic lesions of the pancreas. Pancreatology 2008; 8: 236-51.

2. Basturk O, Coban I, Adsay V. Pancreatic cysts. Pathologic classification, differential diagnosis, and clinical implications. Arch Pathol Lab Med 2009; 133: 423-38.

3. Vázquez-Sequeiros E, Iglesias J. The role of endoscopic ultrasonography in the diagnosis and management of cistyc tumors of the pancreas. En: Domínguez Muñoz E, editor. Clinical pancreatology. Blackwell Publising; 2007. p. 497-503. 
4. Warner EA, Ben-David K, Cendan JC, Behrns KE. Laparoscopic pancreatic surgery: what now and what next? Current Gastroenterology Reports 2009; 11: 128-33.

5. Cienfuegos JA, Rotellar F, Martí Cruchaga P, Valentí V, Zozaya G, Bueno A, et al. Intraductal papillary mucinous neoplasms (IPMN) of the pancreas: clinico-pathologic results. Rev Esp Enferm Dig 2010; 102(5): 314 20 .

6. Hruban RH, Takaori K, Klimstra DS, Adsay NV, Albores-Saavedra J, Biankin AV, et al. An illustrated consensus on the classification of pancreatic intraepithelial neoplasia and intraductal papillary mucinous neoplasms. Am J Surg Pathol 2004; 28: 977-87.

7. Furukawa T, Klöppel G, Volkan Adsay N, Albores-Saavedra J, Fukushima N, Horii A, et al. Classification of types of intraductal papillary-mucinous neoplasm of the pancreas: a consensus study. Virchows Arch 2005; 447: 794-9.

8. Tanaka M, Chari S, Adsay V, Fernandez-del Castillo C, Falconi M, Shimizu M, et al. International consensus guidelines for management of intraductal papillary mucinous neoplasms and mucinous cystic neoplasms of the pancreas. Pancreatology 2006; 6: 17-32. 